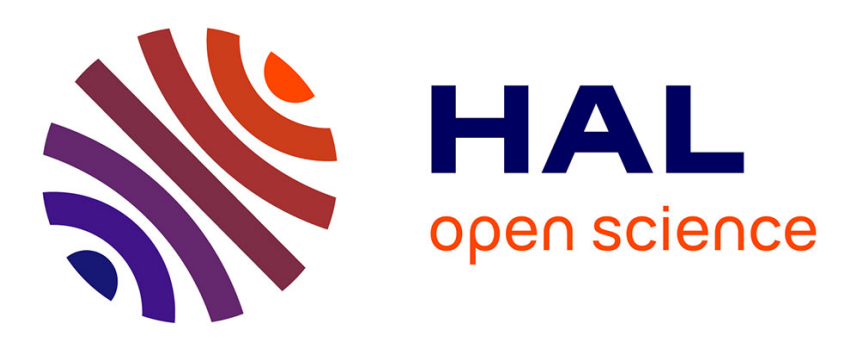

\title{
Utilisation en classe d'un jeu sérieux sur table interactive avec objets tangibles pour favoriser l'activité des élèves : une évaluation comparative en cours préparatoire
}

Sébastien Kubicki, Denis Pasco, Ingrid Arnaud

\section{To cite this version:}

Sébastien Kubicki, Denis Pasco, Ingrid Arnaud. Utilisation en classe d'un jeu sérieux sur table interactive avec objets tangibles pour favoriser l'activité des élèves: une évaluation comparative en cours préparatoire. STICEF (Sciences et Technologies de l'Information et de la Communication pour l'Éducation et la Formation), 2014, Evaluation dans les jeux sérieux, 21, pp.1. hal-01103683

\section{HAL Id: hal-01103683 \\ https://hal.science/hal-01103683}

Submitted on 15 Jan 2015

HAL is a multi-disciplinary open access archive for the deposit and dissemination of scientific research documents, whether they are published or not. The documents may come from teaching and research institutions in France or abroad, or from public or private research centers.
L'archive ouverte pluridisciplinaire HAL, est destinée au dépôt et à la diffusion de documents scientifiques de niveau recherche, publiés ou non, émanant des établissements d'enseignement et de recherche français ou étrangers, des laboratoires publics ou privés. 


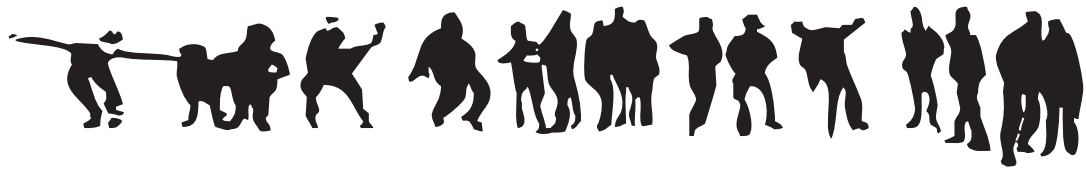

\section{Utilisation en classe d'un jeu sérieux sur table interactive avec objets tangibles pour favoriser l'activité des élèves : une évaluation comparative en cours préparatoire}

Sébastien KUBICKI (ENI Brest, UMR 6285, Lab-STICC, F-29200 Brest, France), Denis PASCO (Université Européenne de Bretagne à Brest, EA 3875, CRÉAD, F-29200 Brest, France), Ingrid ARNAUD (Education Nationale, Circonscription de Brest-Nord, F-29200 Brest, France)

RÉSUMÉ • Dans cette étude, nous évaluons la capacité d'une table interactive avec objets tangibles à favoriser l'activité d'élèves engagés dans un jeu sérieux. Onze élèves de $\mathrm{CP}$ ont participé à cette étude. Chaque groupe a pratiqué en classe la forme traditionnelle du jeu puis, le même jeu sur table interactive. Le temps sur la tâche de chaque élève a été mesuré dans les deux conditions de pratique $\mathrm{du}$ jeu. Les résultats indiquent que le temps que les élèves passent sur la tâche lorsqu'ils pratiquent le jeu sur table interactive est significativement supérieur au temps qu'ils passent sur la tâche dans le jeu en classe. Ils révèlent cependant des différences inter-individuelles significatives. Ils montrent enfin que les activités des élèves sont plutôt de type synchrone dans la pratique du jeu sur table interactive et plutôt de type asynchrone dans la pratique du jeu en classe.

MOTS-CLÉS• Jeux Sérieux; Table Interactive; Interaction Tangible ; Évaluation; Temps sur la tâche ; École primaire ; Étude comparative

ABSTRACT $\bullet$ In this study, we evaluate in which extent a tangible tabletop interface can enhance students' activity involve in playing a serious game. Participants were eleven first grade students. Each group practiced the game in class and then, the same serious game for a tangible tabletop interface. Time on task of each student was measured in the two conditions of practicing the game. Results indicate that the time students spend on task in playing the serious game on a tangible tabletop interface is significantly higher than the time they spend on task when playing the game in class. However, results revealed interindividual differences. Finally, data shown that students' activity is mostly asynchronous in playing the serious game on a tangible tabletop interface while their activity is mostly synchronous in playing the game in class.

KEYWORDS•Serious Games; Interactive Tabletop; Tangible Interaction; Tangible User Interfaces (TUI); Evaluation; Time on task; Elementary school; Comparative study 


\section{Introduction}

La pratique des jeux vidéos constitue une expérience quotidienne des jeunes. Rideout, Foerth et Roberts (Rideout et al., 2010) rapportent qu'en 2009, 60\% des jeunes de 8 à 18 ans pratiquaient des jeux vidéos quotidiennement contre $52 \%$ en 2004 et 38\% en 1999. Selon ce rapport, un jeune entre 8 et 18 ans passerait en moyenne soixante-cinq minutes par jour à jouer à des jeux vidéos.

Au cours des dix dernières années, des chercheurs en éducation ont investi la relation entre les jeux vidéos et l'apprentissage scolaire à travers le développement, la mise en œuvre et l'évaluation de jeux sérieux intégrant un monde numérique (Cruz-Cunha, 2012), (Allain et Szilas, 2012). Klopfer, Osterweil, et Salen (Klopfer et al., 2009) ont défini le jeu comme " une activité volontaire structurée par des règles, avec un résultat défini (gagner/perdre) ou des feedback quantifiables (e.g., des points) qui facilite une comparaison fiable des performances des joueurs» (p. 11). En suivant cette définition, les jeux sérieux constituent des jeux intégrant un monde numérique dont la finalité est l'acquisition de connaissances dans un domaine académique. Ces jeux peuvent être intégrés à un environnement d'apprentissage formel (école ou université, en ligne ou en présentiel), informel (e.g., musées) ou encore, être pratiqués en autonomie par un individu intéressé par l'acquisition de connaissances dans un domaine identifié.

Le plus souvent, les jeux sérieux exposés ci-dessus sont proposés sur des plateformes de type ordinateurs ou consoles de jeu. Cependant, les recherches en Interaction Homme-Machine et les innovations technologiques de ces dix dernières années ont permis de proposer de nouvelles plateformes (e.g. smartphones, tablettes, tables interactives) offrant des interactions nouvelles dites «tactiles», «multitouch» ou encore «tangibles» c'est-à-dire, des interactions «couplant des informations numériques avec des environnements et des objets de la vie quotidienne » selon la définition donnée par Ishii et Ulmer (Ishii et Ullmer, 1997).

Parmi ces nouvelles plateformes, les tables interactives intéressent particulièrement les chercheurs depuis le début des années 90 (Wellner, 1991) en raison de leur grande surface et par conséquent des possibilités qu'elles proposent en terme d'interaction. Les recherches ont par exemple montré que les tables interactives étaient particulièrement adaptées pour la manipulation de photos (Apted et al., 2006), la communication (Piper et Hollan, 2008), le jeu et le divertissement (Tse et al., 2006) ou encore, l'éducation (Khandelwal et Mazalek, 
2007), (Sluis et al., 2004), (Kubicki et al., 2011). Ces tables interactives de part leur grande surface de travail et leur aspect collaboratif semblent en effet particulièrement adaptées pour des applications de type jeux sérieux. Les recherches actuelles visent donc à évaluer l'usage de ces technologies dans le milieu éducatif (AlAgha et al., 2010), les aspects collaboratifs qu'elles engendrent (Martínez et al., 2001), voire de comparer des situations classiques (papier) aux situations numériques sur table (Piper et Hollan, 2009). Cette étude s'inscrit dans ce cadre. Elle vise à éclairer le potentiel des nouvelles plateformes d'interaction à favoriser l'activité d'élèves engagés dans un jeu de mathématiques en comparaison avec leur activité lors de la pratique habituelle de ce jeu en classe.

La présentation de notre étude est organisée de la manière suivante : nous présentons dans un premier temps les travaux relatifs aux jeux sérieux pour l'éducation, puis nous poursuivons dans un second temps par les travaux relatifs à leur usage sur les tables interactives. Nous proposons ensuite deux contributions principales : l'une étant le développement d'une application de type jeux sérieux pour table interactive, la seconde étant son évaluation en milieu scolaire. La troisième section vise donc à présenter d'une façon générale le jeu des tours. Nous exposons dans cette même section la table interactive TangiSense support de nos recherches et enfin le développement et l'adaptation du jeu au format numérique pour la table interactive.

La quatrième section présente notre évaluation en classe en précisant le protocole expérimental, les participants et les mesures envisagées. La cinquième section expose les résultats de nos évaluations basés sur la notion de « temps à la tache». Les résultats sont ensuite discutés dans la section suivante. Enfin, l'article se termine par une conclusion et un ensemble de perspectives de recherche.

\section{Travaux relatifs}

Les travaux exposés dans ce papier s'inscrivent dans deux thèmes proches : les jeux sérieux en éducation et l'utilisation de tables interactives dans la pratique de jeux sérieux. Nous présentons dans cette section les travaux relatifs à ces deux thèmes en rapport avec l'objet de notre étude.

\subsection{Les jeux sérieux et leur efficacité pour l'apprentissage}

Les jeux sérieux sont aujourd'hui investis de manière extensive dans la littérature scientifique dans de nombreux domaines de connaissances comme les sciences (Zuiker, 2008), (Arici, 2009), (Barab et al., 2009), les mathématiques (Ke, 2008), (Kebritchi, 2008), les langues (Peterson, 2010), l'éducation physique 
à travers jeux vidéos actifs (Pasco et al., 2010) ou encore l'histoire (Squire, 2006), (Moshirnia et Israel, 2010).

Par exemple dans le domaine des mathématiques, le jeu sérieux Dimension M consiste, dans un environnement 3D immersif, à accomplir vingt missions dans lesquelles les joueurs apprennent et appliquent des connaissances algébriques (Skal-Gerlock, 2012). Dans le même domaine, Les gardiens de l'oubli est un jeu sérieux développé par JivéCréation (Gastineau et al., 2012) pour l'apprentissage des mathématiques en classe de 4ième. Les joueurs arpentent un univers médiéval dans lequel ils rencontrent des habitants qui ont oublié leurs connaissances et font face à des problèmes à résoudre (e.g., fabriquer des vêtements, concocter des potions et des colorants, affronter des créatures). Les joueurs les aident à résoudre leurs problèmes en mobilisant leurs connaissances en mathématiques.

Dans le domaine des sciences, Quest Atlantis propose une pratique multijoueurs en ligne pour apprendre les sciences (Barab et al., 2009b), (Thomas et al., 2009). Par exemple, dans l'une des unités appelées «Qualité de l'eau du parc de Taïga », les joueurs sont immergés dans un parc national présentant des problèmes écologiques sérieux. Ils se déplacent dans le parc virtuel, interagissent avec d'autres joueurs et des personnages, mobilisent et apprennent des connaissances scientifiques pour comprendre les causes de ces problèmes. Lorsqu'ils disposent d'une compréhension des problèmes, ils sont invités à faire des recommandations pour les solutionner. Ils peuvent ensuite voyager vingt ans dans le futur pour voir le résultat de leurs recommandations.

Enfin, dans le domaine de l'histoire, Civilisation est un jeu de stratégie multijoueurs dans lequel les protagonistes construisent et étendent une civilisation débutant avant l'âge de bronze et se développant jusqu'en 2050 (Squire, 2006), (Moshirnia et Israel, 2010). À travers le développement de leur civilisation, les joueurs apprennent et mobilisent des connaissances historiques. Ils explorent les autres civilisations et prennent des décisions pour développer et protéger leur propre civilisation.

Si les jeux sérieux se sont développés dans les établissements scolaires au cours de ces dernières années, les chercheurs n'ont pas manqué d'interroger leurs effets sur l'apprentissage des élèves. Young et ses collègues (Young et al., 2012) ont conduit une revue de littérature adressant cette question. Sur la base de plus de 300 articles, les auteurs dégagent des évidences empiriques en faveur d'un effet positif des jeux sérieux sur l'apprentissage des élèves dans les langues, en histoire et en éducation physique. Cependant, ils trouvent peu de support dans la littérature en faveur d'un effet positif des jeux sérieux sur l'apprentissage 
des élèves en sciences et en mathématiques. Les auteurs ne pensent pas que la réussite en sciences puisse être liée de manière probante à l'utilisation de jeux sérieux. Ces résultats sont partiellement confirmés dans la revue de littérature de Tobias (Tobias et al., 2011). Si les auteurs identifient que certains jeux sérieux sont susceptibles de mobiliser et de développer des processus cognitifs transférables dans des tâches externes proches, ils soulignent que trop souvent, ils sont développés pour enseigner des contenus pauvres ou la démarche d'apprentissage est intégrée dans le jeu de manière inefficace ce qui contrarie leurs effets sur l'apprentissage des élèves.

L'un des facteurs dégagés par Young (Young et al., 2012) limitant l'efficacité des jeux sérieux pour l'apprentissage est la faiblesse de l'interaction entre le monde numérique et le ou les joueurs. Cette interaction avec le jeu est une condition à l'affordance (i.e. invitation à l'action) qui facilite l'immersion de l'utilisateur dans le jeu et son activité d'apprentissage. Des études récentes se sont penchées sur la problématique de l'interaction entre l'utilisateur et le jeu sérieux en mobilisant des tables interactives.

\subsection{Potentiel des tables interactives pour les jeux sérieux}

Depuis une dizaine d'années maintenant, des chercheurs ont introduit des tables interactives dans la pratique des jeux sérieux. Les tables interactives offrent en effet de nombreux avantages. Côté interaction par exemple, l'usage des deux mains est possible et fortement encouragé sur les tables interactives, ce qui est important car les capacités physiques de la main et du poignet sont riches (Fitzmaurice et al., 1995). D'autre part, les individus peuvent potentiellement se placer n'importe où autour de la table, les entrées sont spatialisées et offrent ainsi le potentiel d'améliorer nettement les capacités de communication. Les artefacts physiques facilitent également l'interaction en rendant l'interface plus directe et surtout plus manipulable (Fitzmaurice et Buxton, 1997). Enfin, la grande majorité de ces tables offrent un espace de travail généreux et permettent à l'utilisateur d'agir tout en parlant et en gardant un oeil sur ce que les autres effectuent comme action (Manches et al., 2009).

Les récentes recherches visent donc à profiter des capacités et avantages offerts par les tables interactives dans la pratique de jeux sérieux. Ces recherches ont notamment fait l'hypothèse que les tables interactives pourraient agir comme une affordance (i.e. une invitation à l'action) pour l'activité des joueurs (Gibson, 1979) et favoriser ainsi leur collaboration. Par exemple, Kelly et ses collègues (Kelly et al., 2010) ont proposé la conception et le développement d'un jeu sérieux (Solar Scramble) sur une table interactive «multitouch » pour des enfants de 5 à 10 ans. L'objectif de l'application était d'apprendre la disposition 
des planètes du système solaire. Ils ont fait évaluer leur jeu sur table interactive par cinq experts de l'enseignement avec des enfants. Ces experts révèlent notamment le potentiel de la pratique multi-joueurs sur table interactive pour favoriser l'activité collaborative des enfants en classe.

Ensuite, Harris et ses collègues (Harris et al., 2009) ont étudié le potentiel d'une table interactive de type «multitouch» à favoriser l'apprentissage collaboratif d'élèves du primaire. Quarante-cinq élèves âgés de 7 à 10 ans ont participé à une étude en classe (21 garçons, 24 filles). Ils ont pratiqué un jeu de déplacements d'objets sur table interactive. Les résultats révèlent que la participation des élèves est significativement supérieure dans la pratique du jeu sur table interactive en modalité «multitouch» qu'en modalité «singletouch » conduisant à des interactions plus collaboratives. Shaer et ses collègues (Shaer et al., 2012) ont aussi étudier la capacité des tables interactives "multitouch » à encourager l'activité collaborative. L'étude en laboratoire de 18 étudiants âgés de 18 à 21 (17 filles, 1 garçon) pratiquant un jeu sérieux (G-nome Surfer 2.0) a révélé les avantages de la table interactive pour l'activité collaborative incluant notamment la réflexion, la participation, l'interaction intuitive et une collaboration effective.

Enfin, Kharrufa et ses collègues (Kharrufa et al., 2010) ont évalué un jeu sérieux sur table interactive «multitouch» liée à une tâche de rédaction collaborative. Les résultats ont montré que l'application favorisait la collaboration, augmentait les discussions en relation avec la tâche dans le groupe et engendrait un haut niveau de réflexion. Une revue de littérature (Higgins et al., 2011) a montré que les tables interactives avaient le potentiel d'offrir des bénéfices significatifs pour l'apprentissage collaboratif.

En complément de ces travaux mobilisant des tables interactives «multitouch », de rares études ont utilisé un couplage entre table interactive et objets tangibles. Pourtant interagir avec une interface tangible (Tangible User Interface, TUI) n'implique pas les mêmes situations d'utilisation qu'une interface graphique classique (Graphical User Interface, GUI). De nombreuses recherches ont démontré les avantages des TUIs comme, par exemple, vivre une expérience plus naturelle et conviviale (Ishii et Ullmer, 1997) et/ou intuitive (Fiebrink et al., 2009). Les TUIs offrent également l'opportunité aux individus d'exercer une manipulation directe qu'on pourrait qualifier d'optimale avec l'interface (Shneiderman, 1981). Plus récemment, la revue de littérature proposée par Zuckerman et Gal-Oz (Zuckerman et Gal-Oz, 2013) vise à proposer une analyse en profondeur des avantages et faiblesses des TUIs vs. GUIs. Leurs résultats montrent que les utilisateurs préfèrent les TUIs ce choix étant motivé par le fait 
que les TUIs proposent des niveaux élevés de stimulation et de plaisir issus de trois propriétés des TUIs : l'interaction physique, la richesses des feedback, et le haut niveau de réalisme. D'un autre côté, les recherches ont montré l'intérêt de la manipulation et de l'usage des objets pour l'apprentissage chez les enfants (i.e., Alibali et diRusso, 1999). Les valeurs pédagogiques de la manipulation d'objets ont par exemple été promues par Maria Montessori : «les enfants construisent leur image mentale du monde, par l'action et les réponses motrices; et, avec la manipulation physique, ils deviennent conscients de la réalité » (Burnett, 1962).

C'est pourquoi certaines recherches visent à tirer profit à la fois des capacités offertes par les tables interactives mais également des capacités offertes par les interfaces tangibles dans la pratique des jeux sérieux. C'est par exemple le cas de l'étude portée par Sluis et ses collègues (Sluis et al., 2004) qui a permis de montrer, grâce à leur application appelée read-it, que le couple table interactive/objets tangibles était intéressant pour l'apprentissage de la lecture chez les enfants de 7 à 9 ans. Marco et ses collègues (Marco et al., 2009), ont évalué ce couplage avec un jeu sérieux favorisant le développement de l'imaginaire (création d'histoire) pour des enfants de 3 à 4 ans. Do-Lenh et ses collègues (Do-Lenh et al., 2010) ont présenté une étude empirique des effets sur la performance et l'apprentissage de l'usage d'une table interactive à interface tangible par des élèves en classe. Les premiers résultats ont montrés que la table interactive améliorait la performance mais qu'elle n'avait pas d'incidence sur l'apprentissage. Plus récemment, Bonnard et al. ont proposé un jeu sérieux sur un système permettant des interactions de type tangible et visant l'apprentissage de la géométrie pour des enfants de 10 à 12 ans (Bonnard et al., 2012). Enfin, Cuendet et ses collègues (Cuendet et al., 2013) ont repris les travaux de Do-Lenh et al. (Do-Lenh et al., 2010) et ont proposé trois environnements d'apprentissage basés sur l'usage de tables interactives à interface tangible en situation réelle en classe. Ces environnements d'apprentissage ont été co-conçus avec des enseignants. Les auteurs présentent dans leur article les caractéristiques qui ont émergé des cycles de co-conception et fournissent un ensemble de recommandations pour la conception de tels environnements d'apprentissage.

Même si un ensemble de travaux existent, les auteurs s'accordent à dire que ce domaine de recherche est encore jeune et que l'on manque d'évidences empiriques notamment par des études en situation réelle en classe pour conclure sur l'efficacité des tables interactives à promouvoir l'apprentissage des élèves dans le cadre de jeux sérieux (Young et al., 2012), (Higgins et al., 2011). Notre étude en classe vise à contribuer à ces évidences empiriques. Elle repose sur l'hypothèse du potentiel des tables interactives avec objets tangibles à offrir 
des conditions de pratique favorisant l'activité d'élèves engagés dans un jeu sérieux comparativement aux conditions de pratique de ce même jeu proposées habituellement en salle de classe. Nous avons évalué en classe la capacité d'une table interactive avec objets tangibles à favoriser l'activité d'élèves (i.e. leur participation physique) engagés dans un jeu sérieux en mathématiques comparativement à la pratique traditionnelle (i.e. support papier) du jeu en classe.

\section{Développement d'un jeu sérieux pour table interactive}

Depuis quelques années maintenant, les ordinateurs et les nouvelles technologies (e.g., tableaux blancs interactifs) sont de plus en plus utilisés dans les écoles (Glover et al., 2005), (Hodge et Anderson, 2007). Nous pensons que les tables interactives pourraient s'intégrer dans les écoles, permettant aux jeunes enfants de travailler de manière ludique seul ou en groupe en leur donnant notamment la possibilité de collaborer. Les élèves pourraient travailler en même temps autour de la table et manipuler des objets avec l'aide de leur enseignant (également présent autour de la table).

\subsection{Contexte de travail}

Une première expérience en école française a été menée en 2011 afin d'évaluer en classe le couplage d'une table interactive (TangiSense) avec objets tangibles avec un jeu sérieux pour la reconnaissance et l'apprentissage des couleurs en maternelle (Kubicki et al., 2011). Cette première expérimentation a permis de déterminer l'impact et la cohérence d'une telle plateforme pour l'apprentissage aussi bien du côté des enfants que du côté des enseignants.

L'objectif de ces recherches est de fournir aux professeurs un ensemble d'outils leur permettant d'exploiter les capacités fournies par les tables interactives. Notre partenariat (formalisé au cours de l'année 2012/2013) avec l'inspection académique de l'Education Nationale nous a permis de mener une étude d'évaluation de l'utilisation d'une table interactive avec objets tangibles dans la pratique d'un jeu sérieux en mathématiques.

Nous proposons dans cette section de présenter dans un premier temps un jeu sérieux appelé jeu des tours habituellement utilisé en classe au format papier par les professeurs des écoles pour un apprentissage en mathématiques puis, la conception et la réalisation de ce jeu pour la table interactive TangiSense afin de le rendre numérique et interactif. 


\subsection{Présentation générale du jeu des tours}

Le jeu des tours est une activité actuellement utilisée par certains professeurs des écoles afin d'appréhender des notions de mathématiques dès le plus jeune âge (Ministère de l'Education Nationale, 2008). Cette activité est issue du livre de Dominique Valentin (Valentin, 2004), professeur de Mathématiques en Institut de Formation des Maîtres (IUFM). Dans son ouvrage, Dominique Valentin propose un ensemble d'activités afin de faire découvrir le monde par les mathématiques aux enfants d'écoles maternelles et primaires.

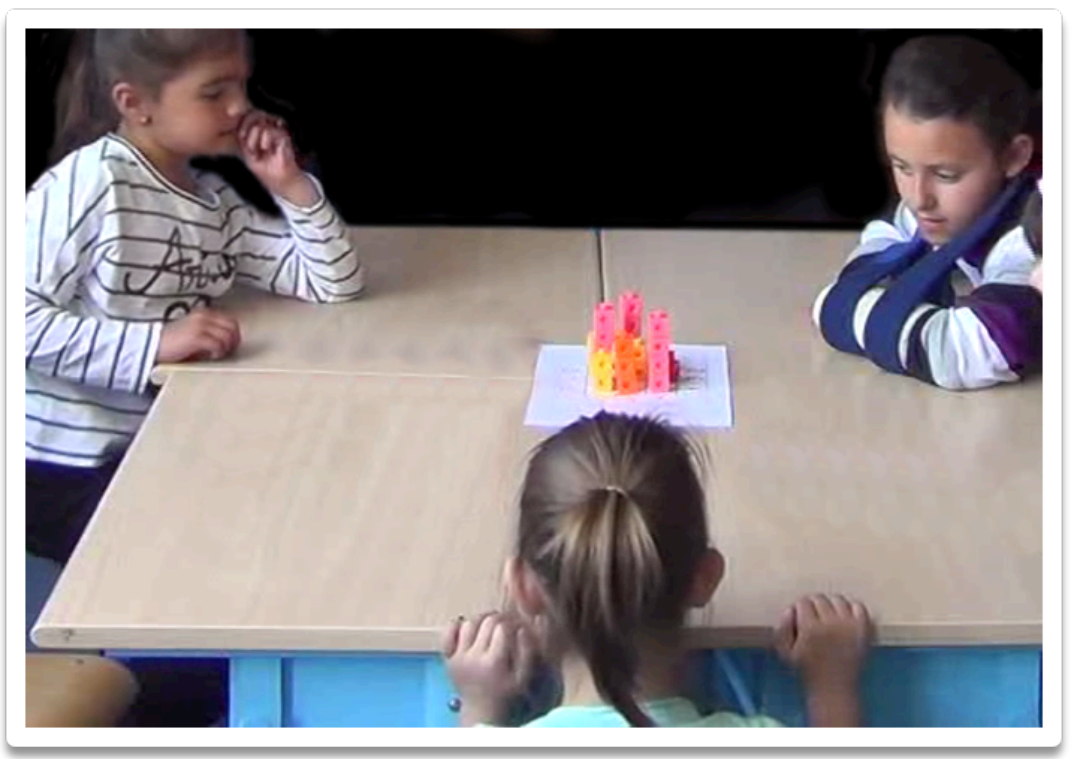

Figure 1 • Exemple de situation où 3 enfants ont un point de vue différent de la disposition des objets sur la table

\subsubsection{Objectifs pédagogiques}

Plusieurs objectifs pédagogiques sont visés par cette activité. Le premier est de faire prendre conscience aux enfants qu'un objet plus grand qu'un autre peut cacher ce dernier. Ainsi selon un angle de vue dans l'espace (e.g., une salle de classe), les enfants doivent se rendre compte que lorsque deux objets ont la même forme mais des hauteurs différentes, l'un peut masquer l'autre. Le second objectif est d'utiliser des informations numériques dans un cadre spatial. Ainsi, si l'on impose la contrainte du nombre d'objets (e.g., quatre objets de même forme mais de tailles différentes (tailles 1, 2, 3 et 4), les enfants doivent appréhender le 
fait que le plus grand des objets (taille 4) peut cacher les trois autres, mais également que l'objet (taille 3) peut cacher les deux objets plus petit (taille 1 et 2) mais pas l'objet de taille 4, etc. Finalement, un autre objectif de cette activité est de faire prendre en compte plusieurs contraintes par les enfants. Ainsi, en reprenant les quatre objets ci-dessus, il est possible - si ceux-ci sont alignés d'imposer deux contraintes aux enfants. Par exemple, selon le point de vue (extrémité «nord» de l'alignement ou extrémité «sud» de l'alignement des objets), il est possible d'imposer que selon le point de vue «nord », l'enfant doive observer trois objets (soit ce placement : taille 1, taille 2, taille 4), mais selon le point de vue "sud» uniquement 2 objets (taille 3, et taille 4) (voir Erreur ! Source du renvoi introuvable.).

\subsubsection{Règles du jeu}

Les objectifs du jeu ayant été présentés, il est maintenant plus facile d'en expliquer les règles relativement simples. Les enfants ont à disposition un ensemble d'objets représentant des tours (assemblage de cubes) de taille différente (hauteur allant de 1 à 5 cubes empilés). Le jeu complet est composé de 17 tours réparties de la façon suivante : une tour de taille 5 puis quatre tours respectivement de taille $4,3,2$ et 1 . Sur la table une feuille de papier représentant une «bandelette » de 5 cases ou une "grille» de $3 \times 3$ ou $4 \times 4$ cases fait office de plateau de jeu. Sur chaque extrémité de la bandelette (ou de la grille) est indiqué un chiffre compris entre 1 et 5 pour la bandelette et entre 1 et 4 pour la grille (voir Figure 2).
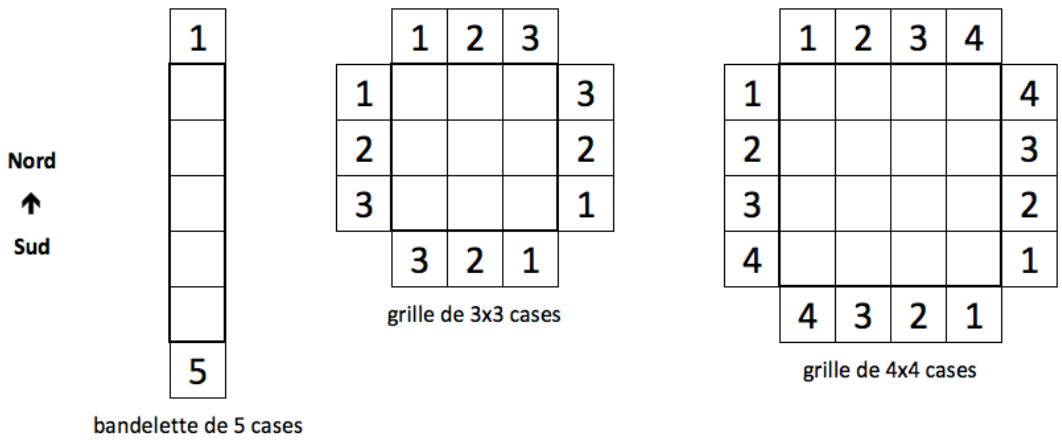

Figure 2 • Exemple de plateaux de jeu

Lors de l'exercice, l'élève doit placer ses tours (qu'il pioche parmi l'ensemble) de manière à respecter les contraintes (chiffres) de chaque extrémité. Chaque chiffre correspond au nombres de tours qu'il sera possible d'apercevoir depuis le point de vue (Nord, Sud) pour la bandelette et (Nord, Sud, Est, Ouest) pour les 
grilles. Ainsi dans l'exemple de la bandelette de la Figure 2, une solution serait de poser les tours respectivement de taille 5, 4, 3, 2, 1 (du Nord au Sud). Selon un point de vue « Nord », l'élève apercevra une unique tour (la plus grande de taille 5), en revanche selon un point de vue «Sud », l'élève apercevra cinq tours (de la plus petite à la plus grande). L'exercice reste le même pour les grilles sachant qu'il faut respecter les contraintes pour les lignes et les colonnes.

Notons que le jeu permet deux variantes : (1) autoriser de poser deux tours de même taille sur une même ligne/colonne (plus facile) ou (2) interdire la pose de deux tours de même taille sur une même ligne/colonne (plus difficile). Durant notre évaluation ( $c f . \S 4)$, le professeur des écoles a choisi la variante $\mathrm{n}^{\circ} 1$.

\subsection{Description de la table interactive TangiSense}

La table TangiSense (Kubicki et al., 2009a) (conçue et développée par la société RFIdées) est une table interactive ayant la particularité de proposer uniquement des interactions «tangibles» (a contrario de la majorité des tables interactives qui propose des interactions «tactiles»). Par interactions «tangibles» nous entendons qu'un ensemble d'objets tangibles (physiques) peuvent/doivent être posés sur sa surface. La technologie RFID employée par la table permet la détection de ces objets.

La technologie RFID (Radio Frequency Identification) (Finkenzeller, 2003) fonctionne selon le principe d'un lecteur et d'un émetteur. Les étiquettes RFID (ou tags RFID) sont composées d'une puce pouvant contenir une mémoire, reliée à une antenne réagissant aux ondes radio (Erreur! Source du renvoi introuvable.).

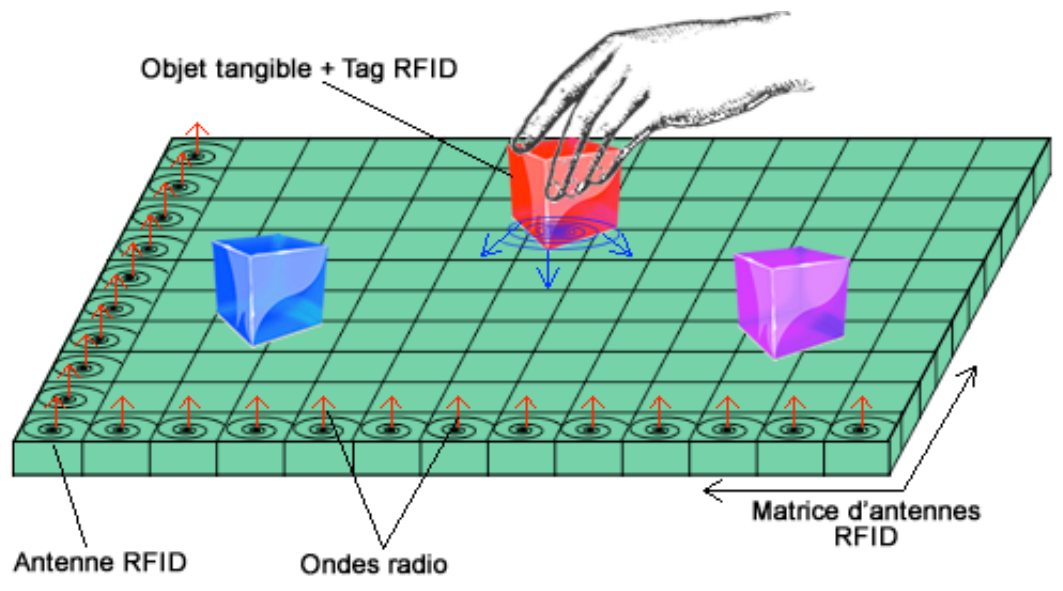




\section{Figure 3 • Schématisation de la technologie RFID employée par la table interactive TangiSense}

Elles sont lues par un lecteur le plus souvent connecté à un ordinateur. La communication entre le composant électronique et le lecteur s'établit par radiofréquence et non par lecture optique (comme par exemple pour la lecture de code barres). Le code barres permet d'identifier une famille de produits tandis que l'étiquette RFID permet l'identification unique d'un objet possédant une étiquette. Remarquons également que les étiquettes RFID peuvent être lues à distance et en parallèle, plusieurs étiquettes pouvant être lues en même temps sur un seul lecteur.

La table dans sa nouvelle version TangiSense 2 (lire (Kubicki et al., 2009b) pour les caractéristiques de la TangiSense 1) est composée de 24 "dalles" contenant chacune 16 antennes RFID ( 4 x 4) de 3,75 cm de côté sur une surface de $90 \mathrm{~cm} * 60 \mathrm{~m}$. Ce principe de dalles ne restreint pas la taille de la table; en ajoutant (ou enlevant) des dalles, il est possible d'augmenter (ou de diminuer) la surface de la table ; la plus petite surface étant la dalle elle-même. Chaque dalle contient son processeur DSP (Digital Signal Processor) de traitement des lectures d'antennes RFID, son multiplexeur d'antennes et son processeur de communication. Les stratégies de lecture sont hiérarchisées et le code est réparti entre le processeur de lecture d'antennes, le processeur en charge du multiplexage et l'ordinateur hôte. Les dalles sont associées entre elles par une interface de contrôle reliée à l'ordinateur hôte par un bus Ethernet. Le temps de réponse obtenu grâce à la communication Ethernet et la lecture RFID offre des performances de vitesse très prometteuses à ce jour. 


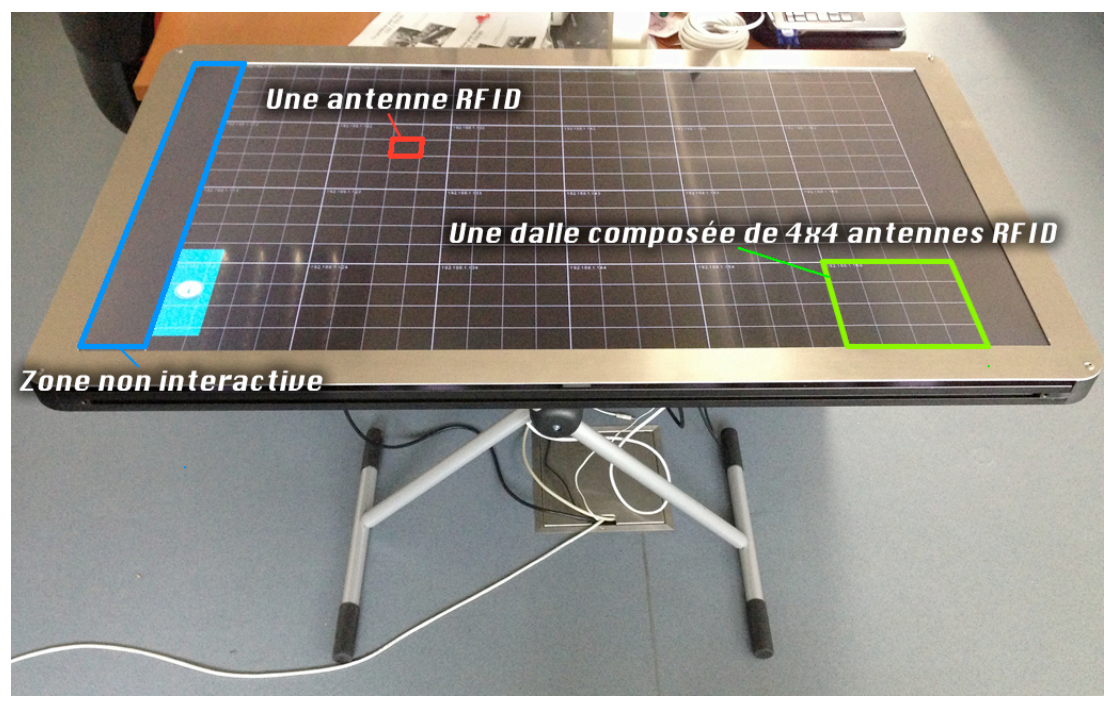

Figure 4 • Présentation de la table interactive TangiSense 2

Dans sa seconde version, la table TangiSense 2 possède un écran LCD de 47 pouces permettant ainsi l'affichage d'objets virtuels avec lesquels interagir grâce aux objets tangibles posés à sa surface. Cette surface intègre de chaque côté de la table une zone «non interactive » d'environ $7 \mathrm{~cm}$, permettant de poser les objets sur la table sans qu'ils ne soient détectés ( $c f$. Erreur! Source du renvoi introuvable.). Enfin, d'un point de vue logiciel, la table interactive TangiSense repose sur un Système Multi-Agents permettant de définir les règles d'interaction entre les agents (objets tangibles/objets virtuels) de l'application (Lebrun, 2013).

\subsection{Développement et adaptation du jeu «papier» pour table interactive}

Le jeu des tours et la table interactive TangiSense, tout deux supports de notre expérimentation ayant été présentés, nous proposons d'introduire dans cette partie l'implémentation du jeu pour la table TangiSense puis la conception des objets nécessaires à l'application.

\subsubsection{Implémentation}

La conception de l'application a fait l'objet d'un projet informatique de fin d'année pour un groupe de 5 étudiants d'une école d'ingénieurs. Le développement de cette première version d'application (nous envisageons un developpement itératif suite à nos expérimentations et remarques que nous 
pourrons obtenir en classes) a été étalé sur une durée de 12 semaines. Notons que la conception a été réalisée en collaboration avec une Conseillère Pédagogique de Circonscription de l'Education Nationale. Celle-ci ayant recommandé le developpement de ce jeu qui est couramment utilisé en classe. Elle a proposé un cahier des charges regroupant l'ensemble des fonctionnalités de bases qui étaient attendus, validé les choix de developpement, et proposé des exemples d'orientation de conception et de validation qui lui semblaient important au regard de son expertise quant à l'usage du jeu et de ses observations de la pratique du jeu en classe par les enseignants. Celle-ci a donc joué le rôle important d'expert pédagogique et d'intermédiaire avec les enseignants dans le développement du jeu.

L'application dans sa première version possède donc un ensemble de fonctionnalité comme :

- une administration de l'application (choix de la classe, saisie/affichage du nom des élèves participants, choix du plateau de jeu)

- une génération automatique des grilles incluant un algorithme de vérification de leur faisabilité

- des algorithmes de correction par ligne ou colonne (aidant les utilisateurs lors de l'exercice) et de validation de grille complète

- une gestion des tags RFID (et par conséquent des objets posés sur la table; nous détaillerons dans la section suivante les aspects de conception des objets)

- Des aspects graphiques (dessin des plateaux, de l'environnement de travail) et sonores (erreur, grille valide, etc.).

L'intégralité de ces fonctionnalités ont permis de rendre l'application fonctionnelle et évaluable en classe. Toutefois, un certain nombre d'améliorations peuvent d'hors et déjà être apportés à cette version suite à notre première expérimentation en classe. Nous discutons de ces améliorations dans les perspectives (§7) de cet article.

\subsubsection{Conception et présentation des objets tangibles}

La table interactive TangiSense permettant d'interagir avec des objets tangibles, il est nécessaire lors du développement d'une nouvelle application de réfléchir aux objets qui seront utilisés puis de les concevoir.

Dans notre cas, les objets devaient respecter certaines contraintes comme par exemple s'approcher le plus possible des objets utilisés actuellement en classe par les enfants et les professeurs, qu'ils soient adaptés aux enfants de 
jeunes âges et par conséquent non dangereux ou coupants et qu'ils ne soient pas métalliques afin d'être correctement détectés par la table.

Dans le cadre de leur projet informatique, les étudiants-ingénieurs du groupe de travail ont exploré et proposé plusieurs pistes pour la conception des objets. Les résultats proposés ont été :

- Une conception en bois, peu coûteuse mais nécessitant quelques heures de travail pour la conception et surtout un respect strict quand à l'éventuel usage de peinture, ou de vernis (afin qu'ils puissent être manipulés en toute sécurité par des enfants).

- Une conception par imprimante 3D des objets. Cette solution est relativement avantageuse côté design puisque n'importe quel objet peut être envisagé. Cependant, les essais et les prototypes d'objets effectués avec le modèle d'imprimante 3D mise à notre disposition ont montré que le temps d'impression était excessivement long pour un unique objet et que le rendu n'était pas à la hauteur des espérances et qu'enfin, le coût pouvait être excessif.

- Une conception à partir cubes ou pièces déjà utilisés par les enfants en classes, comme, par exemple, des LEGO ${ }^{\circledR}$.

C'est finalement pour cette dernière solution que nous avons opté (Figure 5). Les LEGO $^{\circledR}$ sont en effet particulièrement adaptés aux enfants (et utilisés en classe), totalement modulables et par conséquent permettant de concevoir nos objets de la dimension souhaitée. Notons également que le coût de cinq boites de LEGO $^{\circledR}$ (quantité nécessaire pour fabriquer l'intégralité de nos objets) était particulièrement raisonnable (environ $6 €$ par objet conçu).

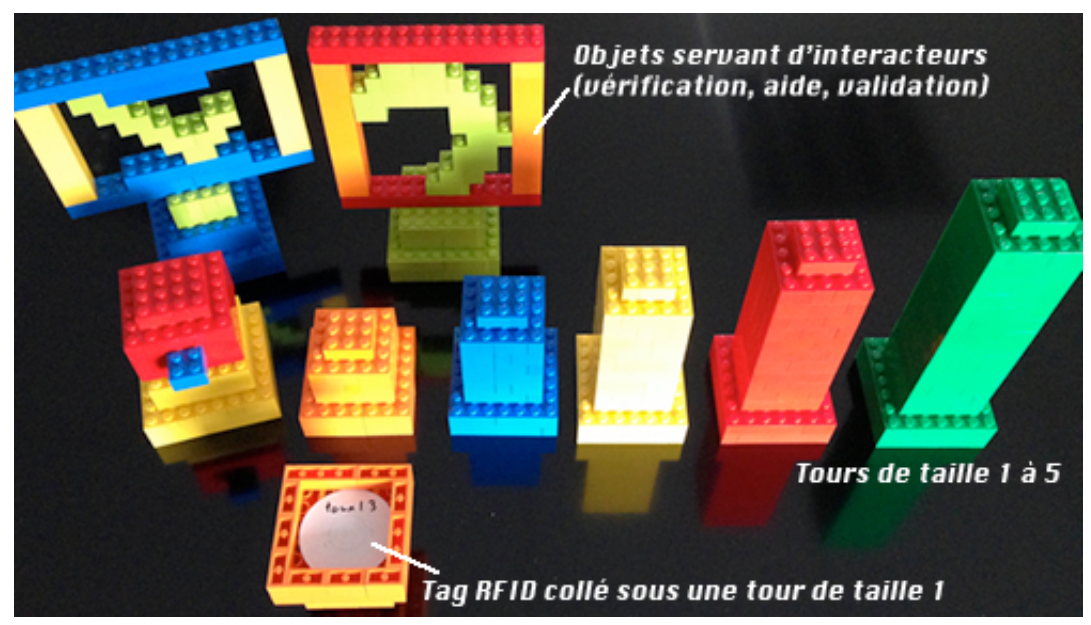




\section{Figure 5 • Les différents objets de l'application conçus en LEGO ${ }^{\circledR}$}

Pour cette application, deux types d'objets ont donc été conçus : (1) les tours de taille 1 à 5 respectant les règles et besoin du jeu initial et (2) des objets « interacteurs» (Lepreux et al., 2012) permettant d'interagir avec la table interactive. Il existe 3 objets de ce type correspondants aux trois tâches que peux effectuer l'enseignant durant la pratique du jeu par des élèves en classe : (1) un objet "vérificateur» (socle jaune, tête rouge sur la Figure 5) permettant de vérifier côté par côté (pour une grille) la position des tours, et d'indiquer à l'enfant ses erreurs et de cibler son attention sur un côté particulier ; (2) un objet « valideur » (socle bleu, « check vert » sur la Figure 5) qui contrôle la bande ou la grille entièrement et qui annonce le résultat à l'enfant (correct / pas correct) ; (3) un objet « aide ${ }^{1}$ » (socle vert, point d'interrogation vert sur la Figure 5) qui permet, lorsque l'enfant en a besoin, d'indiquer directement sur la grille, une ligne (ou colonne) sur laquelle l'enfant doit se focaliser car celle-ci contient une erreur. Notons que sous chaque objet, un tag RFID est collé permettant d'être reconnu par la table interactive (e.g., objet orange en bas de la Figure 5).

\section{Méthode et évaluation}

Dans cette section, nous détaillons la méthode utilisée pour recueillir nos données d'évaluation en présentant les participants à notre étude, le protocole utilisé et le facteur utilisé pour mesurer l'activité des élèves sur le jeu sérieux.

${ }^{1}$ Notons que cet objet n'a pas été utilisé durant notre évaluation afin de ne pas fausser les résultats de comparaison avec l'activité papier durant laquelle les élèves n'étaient pas aidés par l'enseignant. 


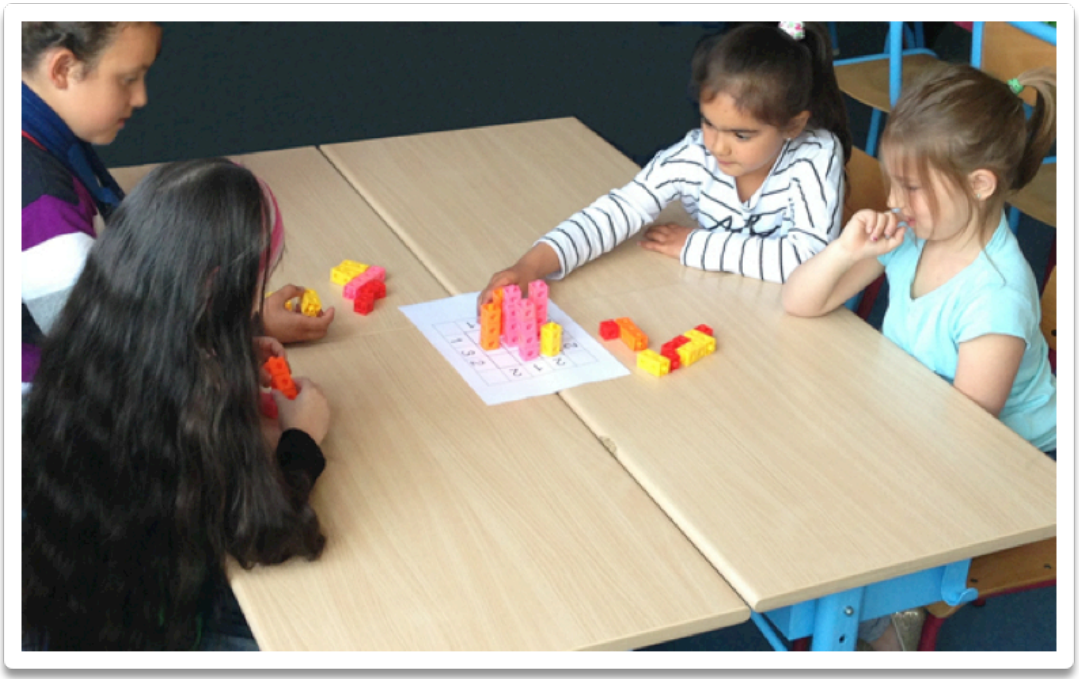

Figure 6 • Quatre élèves autour du jeu au format "papier" en salle de classe

\subsection{Participants et protocole}

Onze élèves de cours préparatoire, 9 filles et 2 garçons (moyenne d'âge : 6 ans 11 mois ; écart-type : 3,9 mois), ont participé à l'étude avec le consentement de leurs parents/représentants légaux. Les élèves ont été répartis de manière aléatoire en deux groupes de quatre élèves et un groupe de trois élèves. Les trois groupes d'élèves ont d'abord pratiqué le jeu en classe une seule fois sous la supervision de leur enseignant dans les conditions dans lesquelles ils le pratiquent habituellement en classe ${ }^{2}$ c'est-à-dire assis autour de tables jointes avec une grille de $3 \times 3$ cases au format A4 papier et le matériel habituellement utilisé (i.e. cubes empilables). Lorsqu'ils pensaient avoir trouvé une solution correcte, les élèves sollicitaient l'enseignant pour vérification. Si cette solution était correcte, l'enseignant la validait et le jeu se terminait. Dans le cas contraire, les élèves cherchaient une solution correcte. La figure 6 présente les conditions de pratique du jeu en classe.

Dans la même journée, les trois groupes d'élèves ont pratiqué le même jeu une seule fois dans les conditions de pratique sur une table interactive avec objets tangibles c'est-à-dire debout autour de la table avec une grille de $3 \times 3$ cases et du matériel adaptés aux dimensions de la table (i.e., tours en $\mathrm{LEGO}^{\circledR}$ ). Après une explication/démonstration par un chercheur du fonctionnement des

${ }^{2}$ Les élèves pratiquent régulièrement ce jeu en classe avec une grille de $3 \times 3$ cases. 
tours et des objets «interacteurs » (phase de familiarisation), les élèves ont cherché une solution correcte au jeu. Lorsqu'ils pensaient l'avoir trouvé, ils utilisaient les objets « vérificateur » et «validateur » pour valider leur réponse. Posés sur des cases spécifiques, ces objets émettaient un bruit d'applaudissements lorsque la réponse était correcte ou un bruit de pleurs dans le cas contraire. Si la réponse était validée, le jeu se terminait. Dans le cas contraire, les élèves cherchaient une solution correcte. La figure 7 présente les conditions de pratique du jeu sur table interactive avec objets tangibles.

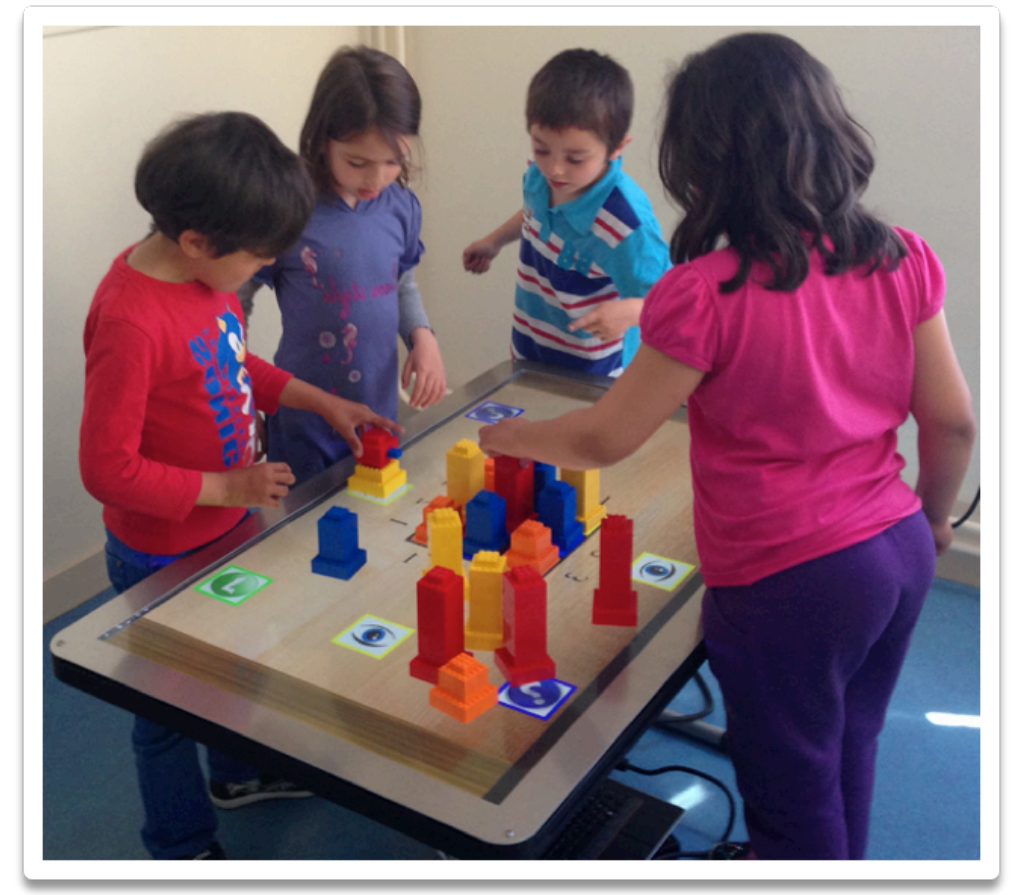

Figure 7 • Quatre élèves travaillant sur le jeu au format numérique

L'activité des trois groupes d'élèves dans les deux conditions de pratique du jeu a été filmée par une caméra placée sur un trépied en surplomb des tables de classe ou de la table interactive.

\subsection{Mesures}

Pour comparer l'activité des élèves sur les vidéos dans la pratique du jeu en classe et sur table interactive avec objets tangibles, nous avons utilisé le facteur du «temps sur la tâche ». Le temps sur la tâche a été défini dans la littérature 
comme la quantité de temps que les élèves passent en étant engagé activement dans une tâche d'apprentissage (Duncheaon et Tierney, 2013). Ce facteur a été avancé dans la littérature comme le facteur le plus important et le plus critique dans l'apprentissage des élèves (Rosenshine et Berliner, 1978), (Stallings, 1980), (Langer, 2001). En effet, plus les élèves passent de temps engagé activement dans une tâche, plus ils ont d'opportunités pour apprendre. Le temps sur la tâche est mesuré à partir de l'activité effective des élèves. Dans notre étude, le temps sur la tâche de chaque élève a été mesuré dans les vidéos par l'activité de placement/déplacement des tours pour trouver une solution au jeu en dehors du temps de familiarisation avec la table interactive. Le temps en seconde a été retenu comme unité de mesure de l'activité des élèves. Deux observateurs ont codé séparément les vidéos pour déterminer de temps sur la tâche de chaque élève dans les deux conditions de pratique du jeu (en classe et sur table interactive avec objets tangibles). Nous avons suivi les recommandations de Rourke et Anderson (Rourke et Anderson, 2004) sur la validité des mesures quantitatives et performé un Kappa de Fleiss (SPSS ${ }^{\circledR}$, v.21) pour déterminer la fidélité inter-codeurs.

\section{Résultats}

Le Kappa de Fleiss calculé sur les codages des vidéos par les deux observateurs indique, selon la table de Landis et Koch (Landis et Kock, 1977), un fort degré d'accord $(\mathrm{K}=.927 ; p<.000)$. Ce résultat a été facilité par le codage du temps sur la tâche de chaque élève à partir de vidéos permettant une analyse plus fine que le codage en situation naturelle obligeant l'observateur à quitter régulièrement les élèves du regard afin de compléter la feuille de codage. L'enregistrement vidéo de l'activité des élèves est à encourager dans ce type de situation pour favoriser la fiabilité des mesures. Nous présentons les résultats de l'évaluation en deux parties. L'une consacrée à la comparaison entre le temps sur la tâche des élèves dans la pratique du jeu en classe sur support papier (traditionnel) et dans sa pratique sur table interactive (numérique). L'autre centrée sur l'évolution chronologique de ce temps sur la tâche dans les deux conditions de pratique du jeu.

\subsection{Temps sur la tâche dans le jeu en classe et sur table interactive}

Le tableau 1 ci-dessous présente le temps total sur la tâche de chaque groupe d'élèves et de l'ensemble des élèves dans la situation de jeu en classe (traditionnel) et dans la situation de jeu sur table interactive avec objets tangibles (numérique). 


\begin{tabular}{|c|c|c|}
\hline \multirow[t]{2}{*}{ Élèves } & \multicolumn{2}{|c|}{ Temps en secondes (\% du temps total d'activité) } \\
\hline & Traditionnel & Numérique \\
\hline 0101 & $3 \quad(1.29)$ & $11(4.36)$ \\
\hline 0102 & $62(26.72)$ & $82(32.54)$ \\
\hline 0103 & $11(4.74)$ & $34 \quad(13.49)$ \\
\hline 0104 & $9 \quad(3.88)$ & $111(44.04)$ \\
\hline Total Groupe 1 & $85 *(36.63)$ & $238^{*}(94.44)$ \\
\hline 0201 & $109(17.60)$ & $125(21.77)$ \\
\hline 0202 & $20 \quad(3.23)$ & $36 \quad(6.27)$ \\
\hline 0203 & $22(3.55)$ & 88 (15.33) \\
\hline 0204 & 21 (3.39) & $35(6.09)$ \\
\hline Total Groupe 2 & $172^{*}(27.78)$ & $284^{*}(49.47)$ \\
\hline 0301 & $29(5.53)$ & 57 (8.79) \\
\hline 0302 & $0 \quad(0.00)$ & $7 \quad(1.08)$ \\
\hline 0303 & $24 \quad(4.58)$ & $62(9.56)$ \\
\hline Total Groupe 3 & $53^{*}(10.11)$ & $126^{*}(19.44)$ \\
\hline Ensemble & $310^{*}(22.54)$ & $648^{*}(43.96)$ \\
\hline
\end{tabular}

Tableau 1 • Temps sur la tâche en classe et sur table interactive

Le temps que les élèves passent sur la tâche lorsqu'ils pratiquent le jeu sur table interactive est significativement supérieur au temps qu'ils passent sur la tâche dans le jeu en classe pour les trois groupes. Les élèves passent en moyenne sur la tâche $22.54 \%$ du temps total d'activité lorsqu'ils pratiquent le jeu en classe contre $43.96 \%$ lorsqu'ils le pratiquent sur table interactive soit presque le double. Sur l'ensemble des élèves, le test Wilcoxon sur échantillons liés indique que le temps passé sur la tâche sur table interactive (M=8.91; ET=10.92) est supérieur au temps passé sur la tâche lors du jeu en classe $(\mathrm{M}=3.89 ; \mathrm{E}-\mathrm{T}=8.78)$, $\mathrm{Z}=4.253, p<.000$.

On observe aussi que, si tous les élèves voient leur temps sur la tâche augmenter dans la situation de jeu sur table numérique, cette augmentation n'est pas identique pour tous. L'élève 0104 voit ainsi son temps sur la tâche augmenter de plus de $40 \%$ sur table interactive passant de 9 secondes $(3,88 \%$ du temps total d'activité) dans la classe à 111 secondes $(44,04 \%$ du temps total d'activité) sur table numérique. Une telle augmentation ne se retrouve pas pour d'autres élèves. Par exemple, l'élève 0101 passe de 3 secondes de temps sur la tâche en classe à 11 secondes sur table numérique tandis que l'élève 0302 n'a aucun temps sur la tâche dans le jeu en classe et uniquement 7 secondes sur table interactive. Les élèves 0102, 0201 et 0301 qui étaient les élèves les plus actifs sur le jeu en classe dans les groupes 1, 2 et 3 sont aussi ceux qui restent les plus actifs dans le jeu sur table interactive. 


\section{2. Évolution chronologique du temps sur la tâche dans les deux situations de mesure}

Le graphique 1 ci-dessous présente une représentation schématique de l'évolution chronologique du temps sur la tâche de chaque élève de chaque groupe lors du jeu en classe et sur table interactive

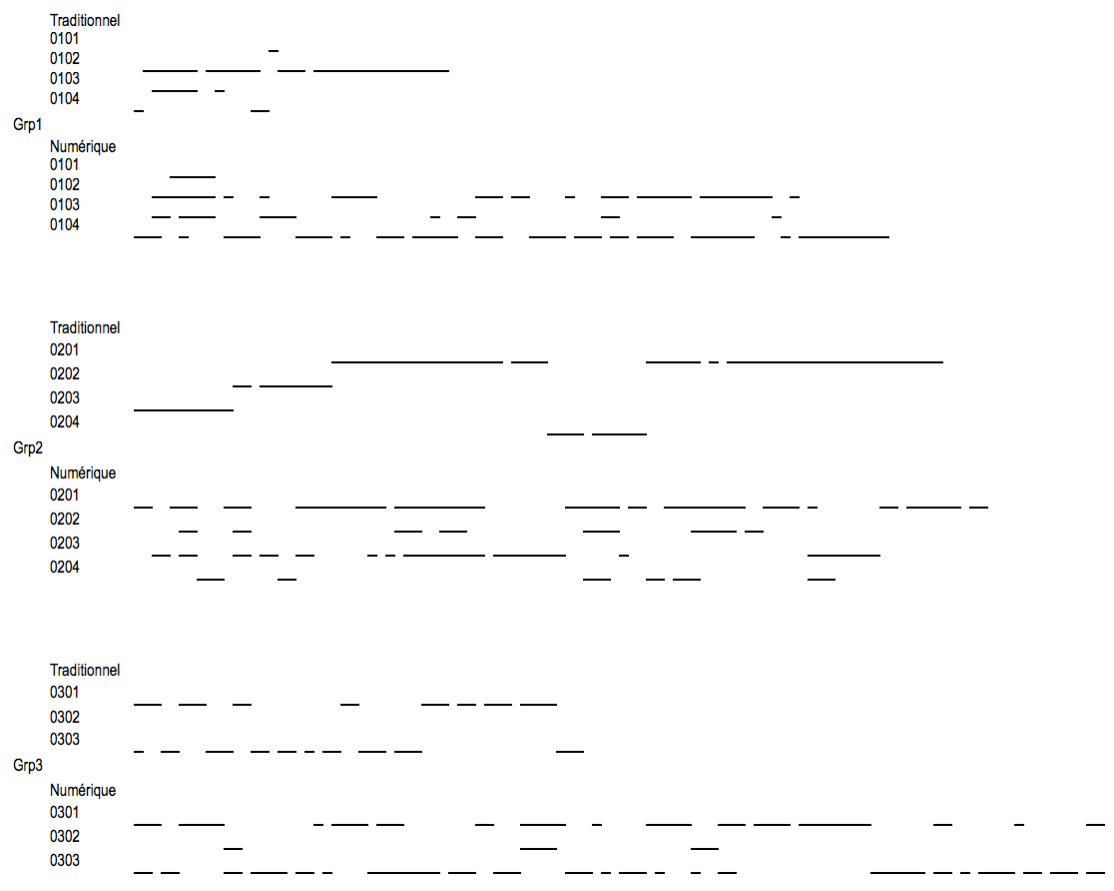

\section{Graphique 1 • évolution chronologique comparée du temps sur la tâche de} chaque groupe d'élèves dans le jeu en classe et sur table interactive

On observe que l'activité des élèves dans la pratique traditionnelle du jeu en classe est majoritairement de type asynchrone c'est-à-dire qu'ils manipulent les tours à tour de rôle pour trouver une solution au jeu. Quelques activités se recoupent pour le groupe 1 tandis que les élèves des groupes 2 et 3 présentent des activités parfaitement asynchrones dans la situation de jeu en classe. Au contraire, dans la pratique du jeu sur table interactive, on observe que, s'il existe des épisodes asynchrones, l'activité des élèves dans les groupes 1 et 2 est majoritairement de type synchrone c'est-à-dire qu'ils manipulent les tours en même temps pour trouver une solution au jeu. Pour le groupe 3 cependant, les activités restent majoritairement synchrones. 


\section{Discussion}

L'objectif de cette étude était d'évaluer l'effet de l'introduction en classe d'une table interactive avec objets tangibles comme outil d'interaction entre des élèves et un jeu sérieux en mathématiques. Onze élèves de cours préparatoire ont participé à cette étude. Ils ont été répartis en trois groupes de manière aléatoire. Ils ont pratiqué le jeu en classe puis, dans la même journée, le jeu sur table interactive. Leur activité dans les deux situations a été enregistrée à l'aide d'une caméra numérique. Nous avons comparé sur vidéos le temps que chaque élève passait sur la tâche lors de la pratique traditionnelle du jeu en classe et lors de sa pratique sur table interactive avec objets tangibles. Les résultats indiquent une évolution significative du temps sur la tâche des élèves lors de la pratique du jeu sur table interactive. Ils montrent aussi que cette évolution n'est pas identique pour tous les élèves. Ils révèlent enfin que pour deux groupes d'élèves la table interactive favorise le développement d'activités synchrones sur le jeu. Ces résultats sont discutés au regard de (1) l'affordance de la table interactive pour l'activité des élèves, (2) la nécessité d'accompagner l'introduction d'outils technologiques dans la classe de démarches didactiques et pédagogiques spécifiques et, (3) l'opportunité offerte par la table interactive d'engager les élèves dans une activité d'apprentissage coopérative.

\subsection{Tables interactives avec objets tangibles et activité des élèves}

Notre étude indique que les élèves passent un peu plus de $20 \%$ de leur temps en classe activement engagés à résoudre le problème posé par le jeu. Ce résultat confirme une tendance lourde dans la littérature internationale sur l'utilisation du temps en classe. Même si des évolutions ont marqué l'utilisation du temps des élèves en classe (Duncheon et Tierney, 2013), les résultats des larges études en mathématiques et en lecture conduites dans les années 70 n'ont pas été remis en cause. Les élèves passent, selon les études, entre 20 et $30 \%$ de leur temps en classe engagés activement dans une tâche d'apprentissage. Le reste du temps, ils écoutent l'enseignant, s'organisent pour pratiquer, passent d'une tâche à une autre ou attendent leur tour pour réaliser la tâche. Nos résultats révèlent que le temps passé sur le jeu sur table interactive représente presque le double du temps passé sur le jeu en classe. Cette différence est significative. La table interactive offre plus d'opportunités aux élèves de s'engager activement dans la tâche et d'apprendre de la résolution du problème posé.

Deux éléments principaux peuvent être avancés pour expliquer cette différence. D'une part, la table interactive pourrait agir comme une affordance invitant les élèves à l'action. Le concept d'affordance a été introduit et théorisé par Gibson (Gibson, 1979). Les affordances ont été initialement définies comme 
les propriétés de l'environnement qui sont significatives pour le comportement des animaux. Dans notre étude, la table interactive avec ses objets tangibles apparaît reconnue par les élèves comme un élément significatif de leur environnement d'apprentissage. Cette affordance de la table interactive les invite à l'action se traduisant par une augmentation significative de leur temps sur la tâche. Un ensemble d'études ont confirmé ce phénomène d'affordance des outils technologiques introduits en classe (Gaver, 1991), (O’Rourke, 2005) (Mangenot, 2008). D'autre part, les conditions de pratique du jeu sur table interactive pourraient expliquer ces différences. Notamment, la possibilité de se déplacer librement autour de la table interactive pourrait avoir favorisé les interactions des élèves avec les tours. La table interactive offre des conditions de pratique du jeu favorables à l'engagement des élèves dans la tâche. Ce potentiel constitue une opportunité pour les apprentissages scolaires.

Pour autant, nos résultats montrent que si tous les élèves voient leur temps sur la tâche augmenter dans la situation de jeu sur table interactive, il existe des différences inter-individuelles significatives. Si la table interactive invite les élèves à l'action, tous ne s'y engagent pas dans les mêmes proportions. À côté de gains de temps sur la tâche très significatifs pour certains élèves, d'autres agissent très peu dans le jeu en classe et sur table interactive. Ce résultat pose la question de l'équité de participation des élèves aux technologies introduites en classe. Harris et al. (Harris et al., 2009) dégagent des différences significatives entre filles et garçons dans l'utilisation d'une table interactive selon une modalité multi-touch ou single-touch. L'introduction de la table interactive et plus largement l'introduction d'outils technologiques dans la classe ne peuvent à eux seuls résoudre la problématique de l'engagement actif dans la tâche et de l'apprentissage de tous les élèves. Après avoir recueilli les idées d'enseignants de mathématiques sur la manière d'utiliser des technologies en classe pour favoriser l'apprentissage des élèves (Ruthven et Hennessy, 2002) ont observé la manière dont ces enseignants utilisaient concrètement ces technologies en classe (Ruthven et al., 2008), Ruthven (2009) conclut à la nécessité d'accompagner la mise en œuvre de ces technologies d'un ensemble de ressources incluant des démarches didactiques et pédagogiques spécifiques. En effet, l'introduction de technologies en classe ne peut se passer d'une réflexion didactique sur les contenus à transmettre et leur adaptation aux niveaux des élèves. Mais elle ne peut non plus se passer d'une réflexion sur la manière dont l'enseignant conduit sa classe dans un environnement éducatif nouveau et gère ses interactions avec les élèves de manière à offrir à tous des opportunités égales pour apprendre. Notre étude confirme cette nécessité d'accompagner l'introduction d'outils technologiques en classe de démarches didactiques et pédagogiques spécifiques. 
Enfin, nos résultats révèlent que les activités des élèves sont plutôt de type synchrone dans la pratique du jeu sur table interactive et plutôt de type asynchrone dans la pratique du jeu en classe. Ce caractère asynchrone de l'activité des élèves en classe constitue une tendance lourde des recherches sur les pratiques pédagogiques traditionnelles (Mayer et Alexander, 2011). Même si des évolutions sont constatées, l'organisation et les contraintes du système éducatif conduit le plus souvent les professeurs à adopter un enseignement dans lequel les élèves cherchent individuellement des solutions aux problèmes posés, répondent à tour de rôle aux questions des enseignants et finalement, apprennent seuls. Les méthodes d'apprentissage coopératif sont aujourd'hui largement étudiées et, sous certaines conditions, elles ont été reconnues comme favorisant la réussite des élèves dans plusieurs disciplines scolaires et à plusieurs niveaux d'enseignement (Slavin, 2011). L'apprentissage coopératif réfère à une modalité d'enseignement dans laquelle les enseignants organisent les élèves en petits groupes pour travailler ensemble et aider chacun à apprendre le contenu visé. Ces méthodes efficaces restent encore peu utilisées dans l'enseignement traditionnel. Notre étude montre que la table interactive, en favorisant l'activité synchrone d'élèves en petits groupes, offre une opportunité d'utiliser en classe le potentiel des méthodes d'apprentissage coopératives.

\subsection{Limites}

Si cette étude révèle le potentiel d'une table interactive avec objets tangibles à augmenter le temps des élèves sur la tâche et à favoriser la coopération à la résolution de problèmes, plusieurs limites doivent être identifiées. Tout d'abord, les conditions de recueil de données basées sur la participation volontaire des enseignants n'ont pas permis de travailler avec un plus grand nombre de sujets. Les études futures devront mobiliser un plus grand nombre de sujets pour permettre de stabiliser les premiers résultats de cette étude exploratoire. Ensuite, le temps sur la tâche est l'un des facteurs principaux ressortant dans la littérature en lien avec l'engagement des élèves dans la tâche et leurs apprentissages. Pour autant, d'autres facteurs pourraient venir compléter utilement nos analyses comme le nombre d'erreurs, le nombre d'essais ou encore l'impact de la table interactive avec objets tangibles sur la motivation des élèves à résoudre le problème. Aussi, des différences liées au sexe des élèves pourraient être prises en compte ainsi que l'expérience des utilisateurs. Enfin, les résultats de cette étude sont circonscrits au jeu de mathématiques développé pour la table. Des études complémentaires sont nécessaires mobilisant d'autres applications sur tables interactives avec objets tangibles permettant de disposer d'une vue plus large de la contribution de cette technologie à l'apprentissage en milieu scolaire. 


\section{Conclusions et perspectives}

Si les résultats de cette première étude apparaissent encourageants dans les limites développées ci-dessus, la volonté de montrer que ce type d'outil technologique peut favoriser l'apprentissage des élèves nécessite des études complémentaires. Plusieurs perspectives s'ouvrent ici. Une première en didactique par l'étude du milieu dans lequel les élèves utiliseront cette technologique en rapport avec un contenu d'enseignement et sous la conduite d'un professeur. Une seconde reposerait sur l'étude des processus cognitifs effectivement mobilisés par les élèves dans la résolution du problème permettant d'identifier notamment les changements conceptuels à opérer pour apprendre. La troisième enfin par l'étude des verbalisations des élèves dans le jeu sur table interactive permettant de dégager des évidences empiriques de la coopération dans la résolution du problème posé.

Notre partenariat avec l'Education Nationale étant relativement récent, nous envisageons de poursuivre nos évaluations en classe afin d'effectuer nos recherches directement en milieu naturel, ce qui est un point fort dans les recherches menées actuellement sur les tables interactives et jeux sérieux. Nous envisageons également de poursuivre l'implémentation de notre jeu des tours afin d'y apporter les modifications et améliorations soulevées lors de nos évaluations. Par exemple nous devrons proposer l'inhibition temporaire de la vérification (objet "vérificateur»), les élèves ayant tendance à effectuer une unique modification sur la grille puis à immédiatement vérifier leur choix à l'aide de l'objet. Notons qu'un ensemble déjà conséquent d'améliorations a été soulevé durant nos premières évaluations nous amenant déjà à proposer une version 2 de l'application.

\section{REMERCIEMENTS}

Ce travail de recherche a été partiellement financé par le Ministère de l'Education nationale, de l'Enseignement supérieur et de la Recherche, le Conseil Général du Finistère, Brest Métropole Océane et la ville de Brest. Nous voudrions remercier les étudiants de l'Ecole Nationale d'Ingénieurs de Brest qui ont participé au développement du jeu sur la table interactive. Nous remercions aussi la circonscription Brest-Nord de l'Inspection Académique du Finistère. Enfin, nous tenons à remercier les écoles Paul Langevin et Paul Dukas de Brest (directeurs, enseignants et personnels administratif) ainsi que les parents et les enfants qui ont accepté de participer à cette étude.

\section{BIBLIOGRAPHIE}

(AlAgha et al., 2010) 
ALAGHA I., HATCH A., MA. L., BURD L. (2010). Towards a teacher-centric approach for multi-touch surfaces in classrooms. In ACM International Conference on Interactive Tabletops and Surfaces (ITS '10), ACM Press, New York, NY, USA, p. 187-196.

(Alibali et diRusso, 1999)

ALIBALI M. W., DIRUSSO A. A. (1999). The function of gesture in learning to count: More than keeping track. Cognitive Development, Vol. 14, p. 37-56.

(Allain et Szilas, 2012)

ALLAIN S., SZILAS N. (2012). Exploration de la métalepse dans les «serious games» narratifs. Sciences et Technologies de l'Information et de la Communication pour l'Éducation et la Formation, Vol. 19, p. 1-14.

(Apted et al., 2006)

APTED T., KAY J., QUIGLEY A. (2006). Tabletop Sharing of Digital Photographs for the Elderly. In Proceedings of the SIGCHI Conference on Human Factors in Computing Systems (CHI '06), Rebecca Grinter, Thomas Rodden, Paul Aoki, Ed Cutrell, Robin Jeffries, and Gary Olson (Eds.). ACM Press, New York, NY, USA, p. 781-790.

(Arici, 2009)

ARICI A.D. (2009). Meeting kids at their own game : A comparison of learning and engagement in traditional and three-dimensional MUVE educational-gaming contexts. Dissertation Abstracts International Section A : Humanities and Social Sciences, Vol. 70(2-A), 469.

(Barab et al., 2009)

BARAB S., GOLDSTONE R., ZUIKER S. (2009). Transformational play as a curricular scaffold : Using videogames to support science education. Journal of Science Education Technology, Vol. 18, p. 305-320.

(Barab et al., 2009b)

BARAB S.A, GRESALFI M., INGRAM-GOBLE A., JAMESON E., HICKEY D., AKRAM S., KIZER, S. (2009). Transformational play and Virtual worlds : Worked examples from the Quest Atlantis project. International Journal of Learning and Media, Vol. 1(2). Disponible sur internet: http://ijlm.net/knowinganddoing/10.1162/ijlm.2009.0023 (consulté le 05 juillet 2013).

(Bonnard et al., 2012)

BONNARD Q., JERMANN P., LEGGE A., KAPLAN F., DILLENBOURG P. (2012). Tangible paper interfaces: interpreting pupils' manipulations. In Proceedings of the 2012 ACM international conference on Interactive tabletops and surfaces (ITS '12), New York, NY, USA, p. 133-142.

(Burnet, 1962)

BURNETT A. (1962). Montessori Education Today and Yesterday. The Elementary School Journal, Vol. 63(2), p. 71-77.

(Cruz-Cunha, 2012)

CRUZ-CUNHA M.M. (2012). Handbook of Research on Serious Games as Educational, Business and Research Tools. Hershey, PA : IGI Global.

(Cuendet et al., 2013)

CUENDET S., BONNARD Q., DO-LENH S., DILLENBOURG P. (2013). Designing augmented reality for the classroom, Computers \& Education, Volume 68, October 2013, Pages 557-569, ISSN 0360-1315.

(Do-Lenh et al., 2010)

DO-LENH S., JERMANN P., CUENDET S., ZUFFEREY G., DILLENBOURG P. (2010). Task performance vs. learning outcomes: a study of a tangible user interface in the classroom. In 
Proceedings of the 5th European conference on Technology enhanced learning conference on Sustaining TEL: from innovation to learning and practice (EC-TEL'10), Martin Wolpers, Paul A. Kirschner, Maren Scheffel, Stefanie Lindstaedt, and Vania Dimitrova (Eds.). Springer-Verlag, Berlin, Heidelberg, 78-92.

(Duncheon et Tierney, 2013)

DUNCHEON J.C., TIERNEY W.G. (2013). Changing Conceptions of Time: Implications for Educational Research and Practice. Review of Educational Research, Vol. 83(2), p. 236272.

(Fiebrink et al., 2009)

FIEBRINK R., MORRIS D., MORRIS M. R. (2009). Dynamic mapping of physical controls for tabletop groupware. In CHI '09 : 27th international conference on Human factors in computing systems, pages 471-480, New York, NY, USA, 2009. ACM Press.

(Finkenzeller, 2003)

FINKENZELLER K., (2003). RFID Handbook : Fundamentals and Applications in Contactless Smart Cards and Identification. John Wiley \& Sons, Inc., New York, NY, USA.

(Fitzmaurice et al., 1995)

FITZMAURICE G. W., ISHII H., BUXTON W. A. S. (1995). Bricks : laying the foundations for graspable user interfaces. In $\mathrm{CHI}$ '95 : conference on Human factors in computing systems, pages 442-449, New York, NY, USA, 1995. ACM Press / Addison-Wesley Publishing Co.

(Fitzmaurice et Buxton, 1997)

Fitzmaurice G. W., et Buxton W. (1997). An empirical evaluation of graspable user interfaces : towards specialized, space-multiplexed input. In CHI '97 : conference on Human factors in computing systems, pages 43-50, New York, NY, USA, 1997. ACM Press.

(Gastineau et al., 2012)

GASTINEAU S., LE FUR R., BAUDOUIN C., VEYRET M., COGAN Y., MISIAK G., CHEVAILLIER, P. (2012). Jeu vidéo, plaisir et pédagogie. Retour d'expérience sur la conception d'un jeu vidéo pédagogique et observation de son utilisation expérimentale en classe. Colloque Scientifique Ludovia 2012, Ariège, France. Disponible sur internet.: http://culture.numerique.free.fr/publications/ludo12/gastineau_ludovia_2012.pdf (consulté le 05 juillet 2013).

(Gaver, 1991)

GAVER W.W. (1991). Technology affordances. In Proceedings of the SIGCHI Conference on Human Factors in Computing Systems (CHI '91), Scott P. Robertson, Gary M. Olson, and Judith S. Olson (Eds.). ACM Press, New York, NY, USA, p. 79-84.

(Gibson, 1979)

GIBSON J. J. (1979). The theory of affordances. In R. E. Shaw \& J. Bransford (Eds.), Perceiving, Acting, and Knowing. Hillsdale, NJ: Lawrence Erlbaum Associates.

(Glover et al., 2005)

GLOVER D., MILLER D., AVERIS D., DOOR V. (2005). The interactive whiteboard : a literature survey. Technology, Pedagogy and Education, Vol. 14 (2), p. 155-170.

(Harris et al., 2009)

HARRIS A., RICK J., BONNETT V., YUILL N., FLECK R., MARSHALL P., ROGERS Y. (2009). Around the table: are multiple-touch surfaces better than single-touch for children's collaborative interactions? In Proceedings of the 9th international conference on Computer supported collaborative learning (CSCL'09), Claire O'Malley, Daniel Suthers, Peter Reimann, and Angelique Dimitracopoulou (Eds.), Vol. 1, International Society of the Learning Sciences, p. 335-344.

(Higgins et al., 2011) 
HIGGINS S., MERCIER E., BURD E., HATCH A. (2011). Multi-touch tables and the relationship with collaborative classroom pedagogies: A synthetic review. International Journal of Computer-Supported Collaborative Learning, Vol. 6(4), p. 515-538.

(Hodge et Anderson, 2007)

HODGE S., ANDERSON B., (2007). Teaching and learning with an interactive whiteboard : a teacher's journey. Learning, Media and Technology, Vol. 32 (3), p. 271-282.

(Ishii et Ullmer, 1997)

ISHII H., ULLMER B. (1997). Tangible bits: towards seamless interfaces between people, bits and atoms. In Proceedings of the SIGCHI Conference on Human Factors in Computing Systems, p. 234-241, New York, USA, ACM Press.

(Ke, 2008)

KE F. (2008). A case of computer gaming for math : Engaged learning from gameplay? Computers \& Education, Vol. 51, p. 1609-1620.

(Kebritchi, 2008)

KEBRITCHI M. (2008). Effects of a computer game on mathematics achievement and class motivation: An experimental study. Dissertation Abstracts International Section A : Humanities and Social Sciences, Vol. 69(6-A), p. 2121.

(Kelly et al., 2010)

KELLY A. R., WALLACE J. R., CERAR K., RANDALL N., MCCLELLAND P., MINDY SETO A. (2010). Solar scramble: an educational children's game for collaborative multi-touch digital tabletops. In Proceedings of the 28th ACM International Conference on Design of Communication (SIGDOC '10). ACM Press, New York, NY, USA, p. 27-32.

(Khandelwal et Mazalek, 2007)

KHANDELWAL M, MAZALEK A. (2007). Teaching table: a tangible mentor for pre-k math education. In Proceedings of the 1st international conference on Tangible and embedded interaction (TEI '07). ACM, New York, NY, USA, p. 191-194.

(Kharrufa et al., 2010)

KHARRUFA A., LEAT D., OLIVIER P. (2010). Digital Mysteries: Designing for Learning at the Tabletop. In ACM International Conference on Interactive Tabletops and Surfaces (ITS '10). ACM Press, New York, NY, USA, p. 197-206.

(Klopfer et al., 2009)

KLOPFER E., OSTERWEIL S., SALEN K. (2009). Moving learning games forward. Cambridge, MA : Education Arcade.

(Kubicki et al., 2011)

KUBICKI S., LEPREUX S., KOLSKI C., (2011). Evaluation of an interactive table with tangible objects: Application with children in a classroom, In Procedings of the 2nd Workshop on Child Computer Interaction "UI Technologies and Educational Pedagogy", In Conjunction with CHI 2011 Conference, May 2011, Vancouver, Canada.

(Kubicki et al., 2009a)

KUBICKI, S., LEPREUX, S., LEBRUN, Y., DOS SANTOS, P., KOLSKI C., CAELEN J. (2009). New Human-Computer Interactions Using Tangible Objects: Application on a Digital Tabletop with RFID Technology. In J.A. Jacko (Ed.), Human-Computer Interaction, 13th International Conference, HCI International 2009, San Diego, CA, USA, July 19-24, 2009, Proceedings, Part III, LNCS 5612, Springer-Verlag, pp. 446-455.

(Kubicki et al., 2009b)

KUBICKI S., LEPREUX S., KOLSKI C., PERROT C., CAELEN J. (2009). TangiSense : présentation d'une table interactive avec technologie RFID permettant la manipulation d'objets Tangibles et Traçables, In Proceedings of IHM 2009, 21ème Conférence de 
l'Association Francophone sur l'Interaction Homme-Machine, ACM Press, International Conference Proceedings Series, Octobre 2009, Grenoble, France, p. 351-354.

(Landis et Koch, 1977)

LANDIS J.R., KOCH G.G. (1977). The measurement of observer agreement for categorical data. Biometrics, Vol. 33, p. 159-174.

(Langer, 2001)

LANGER J.A. (2001). Beating the odds: Teaching middle and high school students to read and write well. American Educational Research Journal, Vol. 38, p. 837-880.

(Lebrun, 2013)

LEBRUN Y., ADAM E., MANDIAU R., KOLSKI C. (2013) Interaction Between Tangible and Virtual Agents on Interactive Tables: Principles and Case Study, Procedia Computer Science, Vol. 19, 2013, p. 32-39.

(Lepreux et al., 2012)

LEPREUX S., KUBICKI S., KOLSKI C., CAELEN J. (2012). From Centralized interactive tabletops to Distributed surfaces: the Tangiget concept, International Journal of HumanComputer Interaction, Vol. 28, Issue 11, p. 709-721.

(Manches et al., 2009)

MANCHES A., O'MALLEY C., BENFORD S. (2009). Physical manipulation : evaluating the potential for tangible designs. In TEI '09 : 3rd International Conference on Tangible and Embedded Interaction, pages 77-84, New York, NY, USA, 2009. ACM Press.

(Mangenot, 2008)

MANGENOT F. (2008). Pratiques pédagogiques instrumentées et propriétés des outils : le cas des forums. Sciences et Technologies de l'Information et de la Communication pour l'Éducation et la Formation, Vol. 15, p. 2-20.

(Marco et al., 2009)

MARCO J., CEREZO E., BALDASSARRI S., MAZZONE E., READ J. C. (2009). Bringing tabletop technologies to kindergarten children. In Proceedings of the 23rd British HCI Group Annual Conference on People and Computers: Celebrating People and Technology (BCS-HCI '09). British Computer Society, Swinton, UK, p. 103-111.

(Martínez et al., 2011)

MARTINEZ R., COLLINS A., KAY J., YACEF K. (2011). Who did what? Who said that?: Collaid: an environment for capturing traces of collaborative learning at the tabletop. In Proceedings of the ACM International Conference on Interactive Tabletops and Surfaces (ITS'11). ACM Press, New York, NY, USA, p. 172-181.

(Mayer et Alexander, 2011)

MAYER R.E., ALEXANDER P.A. (2011). Handbook of Research on Learning and Instruction. New York: Routledge.

(Ministère de l'Education Nationale, 2008)

MINISTÈRE DE L'EDUCATION NATIONALE. (2008). Horaires et programmes d'enseignement de l'école primaire. Bulletin Officiel, hors-série $\mathrm{N}^{\circ} 3$ du 19 juin 2008. Disponible sur internet : http://www.education.gouv.fr/bo/2008/hs3/default.htm Consulté le 05 juillet 2013.

(Morshirnia et Israel, 2010)

MOSHIRNIA A., ISRAEL M. (2010). The educational efficacy of distinct information delivery systems in modified video games. Journal of Interactive Learning Research, Vol. 21, p. 383-405.

(0’Rourke, 2005) 
O'ROURKE B. (2005). Form-focused interaction in online tandem learning. CALICO Journal, Vol. 22(3), p. 433-466.

(Pasco et al., 2010)

PASCO D., BOSSARD C., BUCHE C, KERMARREC G. (2010). Utiliser les jeux vidéos actifs pour promouvoir l'activité physique : Une revue de littérature. Sport Science Review, Vol. 20, Issue 1-2, p. 77-93.

(Peterson, 2010)

PETERSON, M. (2010). Computerized games and simulations in computer-assisted language learning : A meta-analysis of research. Simulation \& Gaming, Vol. 41, p. 72-93.

(Piper et Hollan, 2008)

PIPER A.M., HOLLAN J. (2008). Supporting Medical Conversations between Deaf and Hearing Individuals with Tabletop Displays. In Proceedings of the 2008 ACM conference on Computer supported cooperative work (CSCW '08). ACM Press, New York, NY, USA, p. 147156.

(Piper et Hollan, 2009)

PIPER A.M., HOLLAN J. (2009). Tabletop displays for small group study: affordances of paper and digital materials. In Proceedings of the SIGCHI Conference on Human Factors in Computing Systems (CHI '09). ACM Press, New York, NY, USA, p. 1227-1236.

(Rideout et al., 2010)

RIDEOUT V.J., FOERTH U.G., ROBERTS D.F. (2010). GenerationM2 : Media in the lives of 8-to 18-year-olds. Kaiser Family Foundation. Disponible sur internet: http://www.kff.org/entmedia/upload/8010.pdf Consulté le 05 juillet 2013.

(Rosenshine et Berliner, 1978)

ROSENSHINE B.V., BERLINER D.C. (1978). Academic engaged time. British Journal of Teacher Education, Vol. 4(1), p. 3-16.

(Rourke et Anderson, 2004)

ROURKE L., ANDERSON T. (2004). Validity in quantitative content analysis. Educational Technology Research and Development, Vol. 52(1), p. 5-18.

(Ruthven, 2009)

RUTHVEN K. (2009). Towards a naturalistic conceptualisation of technology integration in classroom practice: the example of school mathematics. Éducation \& Didactique, Vol. 3(1), p. 133-152.

(Ruthven et Hennessy, 2002)

RUTHVEN K., HENNESSY S. (2002). A practitioner model of the use of computer-based tools and resources to support mathematics teaching and learning. Educational Studies in Mathematics, Vol. 49(1), p. 47-88.

(Ruthven et al., 2008)

RUTHVEN K., HENNESSY S., DEANEY, R. (2008). Constructions of dynamic geometry: a study of the interpretative flexibility of educational software in classroom practice. Computers and Education, Vol. 51(1), p. 297-317.

(Schneider et al., 2012)

SCHNEIDER B., STRAIT M., MULLER L., ELFENBEIN S., SHAER O., SHEN C. (2012). Phylo-Genie: Engaging Students in Collaborative Tree-Thinking through Tabletop Techniques. In Proceedings of the SIGCHI Conference on Human Factors in Computing Systems (CHI '12). ACM Press, New York, NY, USA, p. 3071-3080.

(Shaer et al., 2012)

SHAER O., STRAIT M., VALDES C., WANG H., FENG T., LINTZ M., FERREIRAE M., GROTE C., TEMPEL K., LIU S. (2012) The design, development, and deployment of a tabletop 
interface for collaborative exploration of genomic data, International Journal of HumanComputer Studies, Vol. 70, Issue 10, October 2012, p. 746-764.

(Shneiderman, 1981)

SHNEIDERMAN B. (1981). Direct manipulation : A step beyond programming languages. Computer, 16(8):57-69, 1981.

(Skal-Gerlock, 2012)

SKAL-GERLOCK S. (2012). A Case Study on the Effects of a Computer Game on a Homeschooled Student's Motivation and Performance in Algebra. Unpublished thesis. California State University, San Marcos. Disponible sur internet: https://csusmdspace.calstate.edu/handle/10211.8/191 Consulté le 05 juillet 2013.

(Slavin, 2011)

SLAVIN R.E. (2011). Instruction based on cooperative learning. In R.E. Mayer \& P.A. Alexander (Eds.). Handbook of Research on Learning and Instruction, New York: Routledge, p. 344-360.

(Sluis et al., 2004)

SLUIS R. J.W., WEEVERS I., VAN SCHIJNDEL C. H. G. J., KOLOS-MAZURYK L., FITRIANIE S., MARTENS J. B. O. S. (2004). Read-It : five-to-seven-year-old children learn to read in a tabletop environment. In IDC '04 : Proceedings of the 2004 conference on Interaction design and children : building a community. ACM Press, p. 73-80.

(Squire, 2006)

SQUIRE K.D. (2006). From content to context : Video games as designed experiences. Educational Researcher, Vol. 35(8), p.19-29.

(Stallings, 1980)

STALLINGS J. (1980). Allocated academic learning time revisited, or beyond time on task. Educational Researcher, Vol. 9(11), p. 11-16.

(Thomas et al., 2009)

THOMAS M.K., BARAB S.A., TUZUN H. (2009). Developing Critical Inplementations of Technology-Rich Innovations: A Cross-Case of the Implementation of Quest Atlantis. Journal of Educational Computing Research, Vol. 41(2), p. 125-153.

(Tobias et al., 2011)

TOBIAS S., FLETCHER J.D., DAI D.Y., WIND A.P. (2011). Review of research on computer games. In S. Tobias et J.D. Fletcher (Eds.), Computer games and instruction. Charlotte, NC : Information Age, p. 127-222.

(Tse et al., 2006)

TSE E., GREENBERG S., SHEN C., FORLINES C. (2006) Multimodal Multiplayer Tabletop Gaming. In Proceedings of the Workshop on Pervasive Games, p. 139-148.

(Valentin, 2004)

VALENTIN D. (2004) Découvrir le monde avec les mathématiques, Hatier.

(Wellner, 1991)

WELLNER P. (1991). The DigitalDesk calculator: tangible manipulation on a desk top display. In Proceedings of the 4th annual ACM symposium on User interface software and technology (UIST '91). ACM, New York, NY, USA, p. 27-33.

(Young et al., 2012)

YOUNG M.F., SLOTA S., CUTTER A.B., JALETTE G., MULLIN G., LAI B., SIMEONI Z., TRAN M., YUKHYMENKO M. (2012). Our Princess Is in Another Castle: A Review of Trends in Serious Gaming for Education. Review of Educational Research, Vol. 82(1), p. 61-89.

(Zuckerman et Gal-Oz, 2013) 
ZUCKERMAN O., GAL-OZ A. (2013). To TUI or not to TUI: Evaluating performance and preference in tangible vs. graphical user interfaces, International Journal of HumanComputer Studies, Volume 71, Issues 7-8, July-August 2013, Pages 803-820, ISSN 10715819.

(Zuiker, 2008)

ZUIKER S.J. (2008). Transforming practice: Designing for limital transitions along trajectories of participation. Dissertation Abstracts International Section A : Humanities and Social Sciences, Vol. 68(7-A), p. 2816.

\section{BIOGRAPHIE DES AUTEURS}

Sébastien Kubicki a effectué sa thèse (11/2008 - 12/2011) en informatique, qui a été soutenue le 2 décembre 2011. Il est Maître de Conférences en Informatique (27ième section) au Lab-STICC/ENI de Brest depuis septembre 2012. Il est membre de l'équipe Interaction Humain Système et Environnement Virtuel (IHSEV) et effectue ses recherches au sein du Centre Européen de Réalité Virtuelle (CERV). Il est spécialisé en Interaction Homme-Machine (IHM), systèmes adaptatifs au contexte et nouvelles interfaces telles que les tables interactives. Il est membre du GT TANGINT et de l'Association Française en Interaction Homme-Machine (AFIHM). E.mail : sebastien.kubicki@enib.fr

Denis Pasco est maître de conférences habilité à diriger des recherches à l'Université Européenne de Bretagne à Brest (UEBB). Il effectue ses travaux dans le domaine des nouvelles technologies et de l'apprentissage au sein du Centre de Recherche sur l'Éducation, les Apprentissages et la Didactique (CRÉAD, EA 3875) et du Centre Européen de Réalité Virtuelle (CERV). Il est membre de l'American Educational Research Association (AERA), de l'Alliance for Health, Physical Education, Recreation and Dance (AAHPERD) et de l'Association Française de Réalité Virtuelle (AFRV). E.mail : denis.pasco@univ-brest.fr

Ingrid Arnaud, professeur des écoles depuis 1998, est conseillère pédagogique sur la Circonscription de Brest-Nord depuis 2012. Elle est membre de la Mission numérique dans le département du Finistère pour l'Education Nationale ; effectue dans les classes des recherches et des expérimentations sur l'impact du numérique (tablettes et tables interactives avec objets tangibles) sur les apprentissages, sur le travail collaboratif des élèves, de même que sur l'évolution des gestes professionnels des enseignants. E.mail : ingrid.arnaud@ac-rennes.fr 